\title{
Clinical Efficacy and Safety of Artemisinin-based Combination Therapies in the Treatment of Uncomplicated Plasmodium Falciparum Malaria in Cameroon: A Systematic Review and Meta-Analysis from Individual Patient Data (2004-2020)
}

Akindeh M. Nji ( $\square$ akindeh@yahoo.com )

Universite de Yaounde I https://orcid.org/0000-0003-0343-1722

Peter Thelma Ngwa Niba Universite de Yaounde I

\section{Palmer Masumbe Netongo}

Universite de Yaounde I

Innocent M. Ali

Universite de Dschang Faculte des Sciences

Randolph Ngwafor

Universite de Yaounde I

Marcel N. Moyeh

University of Buea

Cyrille Mbanwi Mbu'u

Universite de Yaounde I

Dorothy A. Fosah

Universite de Yaounde I

Barbara Atogho-Tiedeu Universite de Yaounde I

Marie-Solange Evehe Universite de Yaounde I

\section{Rosine Dadjeu}

Universite de Yaounde I

Jean Paul Kengne Chedjou

Universite de Yaounde I

Jude D. Bigoga

Universite de Yaounde I

Anthony Ajua

University of Buea 


\section{Eric Achidi}

University of Buea

\section{Esther Tallah}

Universite de Yaounde I

Rose G. F. Leke

Universite de Yaounde I

Alexis Tourgordi

Organisation mondiale de la Sante

\section{Pascal Ringwald}

Organisation mondiale de la Sante

Wilfred F. Mbacham

Universite de Yaounde I

\section{Research Article}

Keywords: Malaria, efficacy, safety, Plasmodium falciparum, artemisinin-based combination therapies, systematic review, meta-analysis

Posted Date: July 22nd, 2020

DOI: https://doi.org/10.21203/rs.3.rs-45427/v1

License: (c) (i) This work is licensed under a Creative Commons Attribution 4.0 International License. Read Full License 


\section{Abstract}

Background: Cameroon remains a high malaria endemic country. The rapid emergence and spread of Plasmodium falciparum resistant parasites compelled the World Health Organisation (WHO) to recommend the change from monotherapies to artemisinin-based combination therapies (ACTs). This study aimed to assess the clinical efficacy and safety of artemisinin-based combination therapies in the treatment of uncomplicated Plasmodium falciparum malaria in Cameroon from 2004 to 2020.

Methods: The preferred reporting items for systematic review and meta-analysis (PRISMA) statement were adopted for the selection of studies. The heterogeneity of the included studies was determined using Cochrane $\mathrm{Q}$ and the $\mathrm{I}^{2}$. The random effects model was used as standard to combine studies showing heterogeneity of Cochrane $\mathrm{Q}$ with $P<0.10$ and $\mathrm{I}^{2}>50$.

Results: Out of the 4,920 articles and unpublished datasets screened, 16 records with a sample size of 3,737 participants on 8 generic ACTs fulfilled the inclusion criteria. The per protocol (PP) analysis pooled efficacy of the ACTs was $97.9 \%(95 \% \mathrm{Cl}, 97.2-98.7, \quad P<0.01)$. Sub-group analyses were performed for ASAQ, AL and DHAP. The aggregated efficacies of ASAQ, AL and DHAP were $97.5 \%$ (95 \% Cl, 96.3-98.8, $\mathrm{P}<0.01), 99.4 \%$ (95\% Cl, 98.6-100.0, $\mathrm{P}=0.39), 98.0$ \% (95\% Cl, 96.3-99.7, $\mathrm{P}=0.28$ ) respectively. The pooled efficacies were above the WHO minimum benchmark of $90.0 \%$. The ACTs are well tolerated and common adverse events reported were asthenia, diarrhoea, abdominal pain, anorexia, nausea and vomiting, headache and dizziness.

Conclusion: This study reported a high pooled efficacy for all ACTs. AL and DHAP were found to have higher cumulative efficacies than ASAQ. The ACTs are still efficacious and well tolerated for the treatment of malaria in Cameroon. However, there is need for continuous monitoring of efficacy of ACTs despite the high cure rates as resistance seems inevitable.

\section{Background}

Malaria is a major public health disease in Cameroon with Plasmodium falciparum responsible for most of the cases [1]. In 2018, malaria accounted for 228 million cases and 405,000 related deaths worldwide (1). Early diagnosis and treatment of clinical cases remain the main tools for the control of malaria in several regions of Africa [2]. In Cameroon, until 2002, the first-line recommended therapy for uncomplicated falciparum malaria was chloroquine (CQ) and later amodiaquine (AQ) monotherapy between 2002 and 2004 [3]. However, this policy was threatened by the emergence and spread of Plasmodium falciparum resistance to chloroquine (CQ), amodiaquine (AQ) and sulphadoxinepyrimethamine (SP) in most malaria endemic countries. This resulted in major challenges to malaria control in sub-Saharan Africa $[2,4]$. Parasite resistance to monotherapies compelled WHO to recommend combination of dual or triple therapy, which combines molecules with independent modes of action or distinct target enzymes [5]. Therefore, WHO recommended the adaptation and implementation of artemisinin-based combination therapies (ACTs) as the first-line treatment for malaria since early 2000 in 
most countries with endemic $P$. falciparum malaria (6). Five ACTs are currently recommended by WHO for the treatment of uncomplicated Plasmodium falciparum infection: artemether-lumefantrine (AL), artesunate-amodiaquine (ASAQ), artesunate-mefloquine (ASMQ), artesunate-sulphadoxinepyrimethamine (ASSP), dihydroartemisinin-piperaquine (DHAP) [7]. The basis for the use of ACT relies on the rapid reduction of the parasite biomass, reduction of transmission (reducing gametocytes), protection of partner drug against resistance, and rapid fever reduction [8]. In January 2004, Cameroon officially aligned with the recommendations of WHO and adopted artesunate-amodiaquine (ASAQ-75\%) and later included artemether-lumefantrine (AL-25\%) in 2006 as first-line treatment of uncomplicated malaria [9]. These drugs are distributed by those proportions in public facilities, while AL is relatively predominant within the private health facilities and vendors [10]. A recent network meta-analysis (NMA) study on ACTs in Cameroon revealed that AL was more efficacious than ASAQ [11]. The advantage of the NMA approach is that it provides estimates of the effect of each intervention relative to each other [12]. However, the method adopted for the NMA study is not without limitations namely: non-inclusion of observational studies, use of intention-to-treat (ITT) approach and the non-adoption of the individual patient data (IPD) in quantitative syntheses [11]. The adoption of multiple first line ACTs has the potential to delay the emergence of parasites resistant to the anti-malarials [13]. This study was done to provide additional information on the pooled per protocol (PP) efficacy of ACTs using IPD. Hence, this study aimed to assess the clinical efficacy and safety of Artemisinin-based combination therapies in Cameroon from January 2004 to June 2020 with emphasis on ACTs adopted for first-line treatment of uncomplicated falciparum malaria.

\section{Methods/design}

\section{Searching strategies}

Studies included in this review were selected using the preferred reporting items for systematic review and meta-analysis (PRISMA) statement [14]. A computerised systematic strategy based on key words was used to search articles from PubMed/Medline, Google Scholar, and Science Direct databases. Both interventional and observational studies were retrieved to be included in the review using the following MeSH search terms: 'Cameroon AND malaria AND artemether-lumefantrine', 'Cameroon AND malaria AND artesunate-amodiaquine', 'Cameroon AND malaria AND artesunate-mefloquine', 'Cameroon AND malaria AND dihydroartemisinin-piperaquine', 'Cameroon AND malaria AND artesunate-sulphadoxinepyrimethamine', 'Cameroon AND malaria AND artesunate-atovaquone-proguanil', 'Cameroon AND malaria AND artesunate-sulphamethoxypyrazine-pyrimethamine', and 'Cameroon AND malaria AND artesunatechloguanil-dapsone'. Additional information on Clinical Trials was also obtained from the libraries and from researchers at the Universities of Yaounde I, Buea, Douala, Dschang and Bamenda. The library of the Catholic University of Central Africa based in Yaounde Cameroon was also consulted. Moreover, information was obtained from the OCEAC Bulletin and from the National Malaria Control Programme (NMCP), the Ministry of Public Health annual reports. Furthermore, studies conducted in any of the four sentinel sites in Cameroon were sought from the NMCP or from individual researchers who provided this 
information voluntarily. In addition to published studies, unpublished thesis reports were accessed for inclusion in the study.

\section{Inclusion criteria}

The following studies were included in this systematic review and meta-analysis: original articles of studies that investigated at least one ACT in the treatment of uncomplicated falciparum malaria; studies published that included study periods from January, 2004 to June, 2020; studies written in English or French; all multi-centric studies in which Cameroon was one of the sites were included in this report. The population intervention comparator outcome (PICO) format was used to select and include studies (Additional file 1). The primary objective of this review was to assess the efficacy of ACT measured as treatment success at days 14, 28, 42 or 63 for uncomplicated malaria caused by Plasmodium falciparum, while the frequency of adverse events (AEs) was the secondary objective. AEs were defined as 'signs and symptoms that first occurred or became more severe post-treatment' or 'as a sign, symptom, or abnormal laboratory value not present on day 0 , but which occurred during follow-up, or was present on day 0 but became worse during follow up'. Serious adverse events were defined according to International Conference on Harmonisation (ICH) guidelines. Studies included in this review are shown in Additional file 1.

\section{Non-inclusion criteria}

The following papers were excluded from this systematic review and meta-analysis: studies that used artemisinin monotherapies or non-artemisinin monotherapies; non-artemisinin combination therapies; studies that assessed malaria treatment outcomes at times less than 14 days and studies with PCR unadjusted cure rates. Studies that were excluded from this review are shown in Additional file 1.

\section{Review process}

All of the research articles identified from searches of the electronic databases were screened for eligibility based on title and abstract. Ineligible articles and duplicates were eventually removed using Zotero standalone software version 5.0.56. Full-length articles of the selected studies were read to confirm for fulfilling of the inclusion criteria before data extraction began. Two reviewers (PTNN and $\mathrm{CMM}$ ) independently screened the titles and abstracts to identify potentially eligible studies and data extracted from full-length articles that fulfilled the inclusion criteria (Figure 1). Discrepancies were resolved by mutual consent after discussion and independent review from the third researcher (AMN). WFM reviewed the whole process.

\section{Data extraction procedure}


Data on the types of study design (observational versus interventional), year the studies were conducted, duration of study, and geographic location of the study area was first extracted. Participants' age ranges were then extracted. Finally, data regarding the types of anti-malarial treatments, treatment outcome measures (including treatment success rates, treatment failure rates), treatment duration, and adverse events (AEs) were extracted to be included in the systematic review and meta-analysis.

\section{Methodological quality assessment and sensitivity analysis}

The quality of the reviewed studies was assessed through sensitivity analysis, which classified the included studies into high quality and low quality according to modified Jadad scale for randomised controlled trials (RCTs) [15] and the strengthening the reporting of observational studies in epidemiology (STROBE) statement for observational studies [16]. Modified Jadad scale assesses the quality of a trial with the range from 0 to 8 (randomisation and its appropriate use, blinding and its appropriate use, withdrawals and dropouts, description of inclusion and exclusion criteria, assessment of adverse effects, and description of statistical analysis). The score for the modified Jadad scale range of 0-3 represents low or poor quality and score ranges of 4-8 represents good to excellent quality. The observational studies were categorised as low quality with a score under $75 \%$ of the STROBE checklist and high quality with a score over $75 \%$ of the STROBE checklist. The reviewers independently assessed the quality of the methodology of included studies.

\section{Assessment of treatment outcomes}

Treatment outcome was assessed as treatment failure and treatment success. The outcomes of all the studies included in this review were assessed and analysed on the $14^{\text {th }}, 28^{\text {th }}, 42^{\text {nd }}$ and $63^{\text {rd }}$ day of treatment. Treatment failure included: early treatment failure (ETF), late parasitological failure (LPF) and late clinical failure (LCF). The indicator for treatment success was adequate clinical and parasitological response (ACPR). ACPR was defined as absence of parasitaemia by the end of treatment (days 14, 28, 42, 63) irrespective of axillary temperature without previously meeting any of the criteria for early treatment failure or late clinical failure or late parasitological failure (17-20). The treatment success was defined based on PCR genotyping according to current World Health Organisation (WHO) recommendation.

\section{Publication bias}

Publication bias was assessed using funnel plot with the standard error of each study plotted against its effect size (Additional file 2). The Egger test was also used to assess publication bias.

\section{Data analysis and heterogeneity assessment}


The traditional meta-analysis that estimates a common effect of the same intervention A, B and C by pooling individual patient data (IPD) from various studies was adopted. The $\mathrm{R}$ software package version 3.5.2 was used to carry out all the meta-analyses of malaria treatment efficacy. The heterogeneity of the included studies was investigated using Cochrane $Q$ and the $I^{2}$. The random effects model was used as standard to combine studies showing heterogeneity of Cochrane $\mathrm{Q}$ with $P<0.10$ and $\mathrm{I}^{2}>50$ [21]. Heterogeneity using was classified as low (0-49\%), moderate (50-74\%) and high (75-100\%).

\section{Ethical considerations}

The PRISMA guideline recommendations were used and strictly followed to carry out this systematic review and meta-analysis. Ethical approval is not recommended and was not needed since it is a systematic review and meta-analysis.

\section{Results}

\section{Study identification and selection process}

A computerised systematic strategy was used to identify and screen articles from PubMed, Google Scholar, and Science Direct databases for eligibility (Figure 1).

\section{Qualitative synthesis}

The search of studies published from 2004 to 2020 identified 13 articles important to the topic under review [22-34], out of which 10 were RCTs [22-30,33,34] and 3 non-comparative clinical trials without randomisation [31, 32] (Additional file 1). Data from 3 unpublished studies that were conducted by the Cameroon National Malaria Control Programme and independent researchers were also included in this review. A total of 39 studies from 16 records with the same or different ACTs fulfilled the inclusion criteria and were included in this systematic review and meta-analysis with a total sample size of 3,747 participants that ranged from a minimum of 48 patients [22] to a maximum of 255 patients [30]. Treatment outcomes in all studies were evaluated by adopting clinical and parasitological criteria based on WHO guidelines [17-20].

\section{Quantitative synthesis}

A total of 8 generic ACTs were included in this review and meta-analysis. The overall efficacy of the ACTs was $97.9 \%(95 \% \mathrm{Cl}, 97.2-98.7)$ and heterogeneity, $Q=82.3, \mathrm{l}^{2}=61 \%, \mathrm{X}^{2}=97.9(\mathrm{df}=38), \mathrm{P}<0.01$. Most of efficacies were above the WHO limit of $90 \%$ with the exceptions of AS-CD and AS-SP with values of 84.5 $\%$ and $86.0 \%$ respectively. The most common ACTs included DHAP, AL, and ASAQ with a total of $4,9,16$ and studies respectively (Figure 2). 
Sub-group analyses were carried out for ASAQ, AL and DHAP. The pooled efficacy of ASAQ was $97.5 \%$ (95\% Cl, 96.3-98.8,) with heterogeneity, $Q=37.7, \mathrm{I}^{2}=60 \%, \mathrm{X}^{2}=37.7(\mathrm{df}=15), \mathrm{P}<0.01$ (Figure 3 ). The overall efficacy of $A L$ was $99.4 \%(95 \% \mathrm{Cl}, 98.6-100.0)$ with heterogeneity, $Q=5.4, I^{2}=6 \%, X^{2}=8.5(\mathrm{df}=8), P=0.39$ (Figure 4). The aggregated efficacy of DHAP was $98.0 \%(95 \% \mathrm{Cl}, 96.3-99.7)$ with heterogeneity, $\mathrm{Q}=3.8$, $\mathrm{I}^{2}=22 \%, \mathrm{X}^{2}=3.8(\mathrm{df}=3), \mathrm{P}=0.28$ (Figure 5 ).

\section{Evolution in the efficacies of ASAQ and AL from 2004 to 2020}

A decline in Artesunate-Amodiaquine (ASAQ) efficacy was observed over time from an original $100 \%$ to a current value of $91.8 \%$ (Figure 6 ). The PCR adjusted cure rate of artemether-lumefantrine reduced from an original of $100 \%$ to a current value of $96.7 \%$ (Figure 6 ).

The studies were grouped according to the Littoral, Forest, Savanna and Sahel-Savannah ecological zones similar to the four ecological facets defined by Cameroon's National Malaria Control Programme. It was demonstrated that the efficacy of ASAQ declined faster in some ecological zones when compared to others. The greatest deep in ASAQ was noticed in the Littoral ecological zone (Figure 7).

\section{Fever and parasite clearance by ACTs}

Fever temperature measurements were mostly axillary and on average were above $38^{\circ} \mathrm{C}$. Fever was rapidly cleared by the ACTs with low proportions with fever on D1 of $17 \%$ and $5 \%$ by D3 (Additional file 5). Some authors did not measure fever clearance on subsequent days post drug administration and only choose D3 for this clinical measurement.

Starting parasitaemia with geometric mean densities varied between 3800 to 42000 parasites per ml. Parasite clearance was very rapid sometimes dropping to 7-8 \% on D1 but on average stayed at 30-50\%. Parasite clearance times were similar across study arms in most studies. Nji et al., observed that there appeared to be a study site effect on parasite clearance between Garoua and Mutengene [30]. Although not significant most participants in Mutengene site (75 \%) (Figure 8), did not clear their parasites as measured by microscopy by the end of D1 post-treatment compared to participants in Garoua (32\%). Comparing the parasite clearance time across the different treatment arms and site did not show any significant difference by D3 post-treatment $(p>0.05)$. Overall, the proportion of patients with parasites on D2 was much lower than on D1 and even lower on D3 (Figure 8). Apinjoh et al., found a high occurrence of parasitaemia of $17 \%$ and $35 \%$ respectively in ASSP and ASAQ arms in Buea-Tole on D28 [33] and from which study an efficacy of ASAQ of about $92 \%$ was recorded. The average parasite clearance did not take into account the two studies with outlier figures (Figure 8 and Figure 9). Only two of these studies were interested to have measured parasite levels up till D42 or D63 [30,32]. It should be noted that parasitaemia tended to re-occur between D14-D63. 


\section{Safety and tolerability of ACTs in Cameroon}

A total of $12(76.5 \%)$ articles and unpublished data reported adverse events [23, 24, 26, 28-31, 33]. The ACTs are well tolerated with few adverse events, the most reported across all studies being those of asthenia, diarrhoea, abdominal pain, anorexia, nausea, vomiting, headache and dizziness. Out of 12 studies, 5 reported severe adverse events which included: 1 jaundice, 1 haemoglobulinuria, 3 anaemia, 1 convulsion, 1 severe fatigue, 3 severe malaria and 3 deaths (Additional file 5). A three-arm randomised non-inferiority controlled trial to assess the efficacy and safety of ASAQ, DHAP and AL was conducted in Mutengene and Garoua from 2009 to 2013. The authors demonstrated that the frequency of adverse events such as vomiting, cough, rashes, and anorexia was slightly higher in the groups of participants on ASAQ and DHAP. The drugs did not differ with respect to the type of AEs (all $p$ values $<0.05$ ). Although there was no significant statistical difference $(P=0.09)$ in the occurrence of all AEs when comparing the trial drugs, ASAQ (35.5\%) and DHAP (37.9\%) had higher number of AEs than AL (27.5\%). One serious AE occurred involving a child who experienced severe fatigue after AL ingestion [30].

In another study in Buea, it was observed that while administering ASAQ and SPAS, at least one adverse event (AE) was reported in $69.2 \%(117 / 169)$ of patients in both treatment groups during the posttreatment period that was not present on admission. These were probably related to the study drug and mainly mild or moderate in intensity. The most frequent AEs were cough, dizziness, fatigue, catarrh, and gastrointestinal disorders (nausea, abdominal pain, and diarrhoea). A total of 36 (43.4\%) and 81 (94.2\%) patients in the AS/SP and AS/AQ group, respectively, experienced AEs by day three. By day seven, the number of patients with AEs had reduced to two (4.5\%) and eight (17.7\%) in the AS/SP and AS/AQ groups, respectively [33].

\section{Quality assessment and sensitivity analysis}

The articles included in this review were of high qualities according to modified Jadad scale for randomised controlled trials (RCTs) with values ranging from 5 to 8 (Additional file 3 ) while and the strengthening the reporting of observational studies in epidemiology (STROBE) statement for observational studies with a range of $86 \%$ to $91 \%$ (Additional file 4). There was no need for sub-group analysis because of the high quality of studies included.

\section{Discussion}

The systematic review and meta-analysis aimed to assess the pooled clinical efficacy and Safety of artemisinin-based combination therapies from individual participant data for over 16 years after adoption and use for the treatment of uncomplicated falciparum malaria in Cameroon. We demonstrated that the cure rates of most ACTs were above the WHO minimum limit of $90 \%$ with a cumulative value of $97.9 \%$. This observation is in agreement with cure rate of $98.0 \%$ reported in meta-analysis on ACTs used in Sudan [35], but higher than the efficacy of 92.9\% recorded in Ethiopia [36]. However, 2 studies on 
Artesunate-chloguanil-dapsone (AS-CD) and Artesunate-sulphadoxine-pyrimethamine (AS-SP) failed to meet the WHO, Day 28, PCR-adjusted cut-off of $>90 \%$ efficacy $[27,33]$. Despite the high success rates of ACTs, resistance to anti-malarial drugs poses a major threat globally and if the parasites develop resistance to these anti-malarial regimens, it is inevitable that treatment would be more difficult, unsuccessful and high rates of relapse could be due to multidrug-resistant malaria. Therefore, monitoring the anti-malarial drug efficacy is important to enable early detection of emergence of drug resistance before it spreads to most of the parasite population, similarly to what happened with chloroquine, sulphadoxine-pyrimethamine, and amodiaquine monotherapies in Cameroon [2,37,38]. More so, there is no guarantee how long the currently used anti-malarial drugs will remain effective based on evidence that previous monotherapies were associated with higher rates of treatment failures in $P$. falciparum infected patients [2,39]. This calls for concerted action for search of new alternative anti-malarial drugs to treat malaria in the near future.

The efficacy of ASAQ the first line treatment adopted in 2004 for the treatment of uncomplicated falciparum malaria in Cameroon was $97.5 \%$. Contrary, a slightly lower value of $93.9 \%$ was recorded in a multi-centric study on pooled efficacy of ASAQ in sub-Saharan Africa [40]. Moreover, the efficacy of AL, the parallel first-line drug for the treatment of uncomplicated falciparum malaria in Cameroon was $99.4 \%$. This is in concordance with the $97.3 \%(95.9-98.3 \%, 95 \% \mathrm{Cl})$ treatment success in children with uncomplicated malaria recorded in the pooled analysis of data from seven studies supported by Novartis [41]. This suggests that the results of treatment success with AL in uncomplicated malaria patients in Cameroon are evenly distributed with other high malaria endemic countries. The aggravated efficacy of DHAP was $98.0 \%$. AL and DHAP were found to have higher pooled efficacies than ASAQ. This finding is in agreement with those of different studies on the efficacy of ACTs assessed using the network metaanalysis approach $[11,12,42]$. DHAP has also been shown to be highly efficacious in the clearance of malaria parasites among Human immunodeficiency virus (HIV) patients in Malawi and Mozambique [43]. The standard uncomplicated malaria treatment guideline for Cameroon recommends a three-days administration of ASAQ or AL depending on the weight and age of the patient [44]. Based on the present evidence, it will be advisable to consider DHAP in the treatment of malaria especially among HIV patients where ASAQ and AL may be contraindicated concurrently taking efavirenz-or nevirapine-based antiretroviral therapy. It was observed that the efficacy of ASAQ declined an original value of $100 \%$ to a current value of $91.8 \%$ while that of $A L$ declined from an original value of $100 \%$ to a current value of $96.7 \%$ over time. It was also shown that the efficacy of ASAQ declined faster in some ecological zones when compared to others. The greatest deep in ASAQ was noticed in the Littoral ecological zone. This change could be due to the decline in the efficacy of ASAQ in Buea [33]. However, it is important to note that the study in Buea was among the first studies to evaluate the efficacy and safety of ACTs in real time.

Most studies included in the present review achieved a rapid reduction of fevers and parasitaemia between D0 and D3 of assessment. Majority of these studies treated patients with ASAQ, AL and DHAP. A previous aggregate study on the clinical predictors of early parasitological response to ACTs (ASAQ, AL and DHAP) in African patients with uncomplicated falciparum malaria confirmed the rapid decrease of 
parasite positivity rate (PPR) from 59.7\% (95\% Cl: $54.5-64.9)$ on day 1 to $6.7 \%$ (95\% Cl: 4.8-8.7) on day 2 and $0.9 \%$ (95\% Cl: $0.5--1.2)$ on day 3 [45].

Some studies showed a delayed clearance on D1 with a proportion of $75 \%$ persistence of parasite in Mutengene/Garoua [30] and 35\% parasitaemia on D28 Buea-Tole [33]. It should be recalled that Buea is in a zone which borders with Limbe and Mutengene which are sites for high chloroquine-resistance [2, 46].

The ACTs, ASAQ, AL and DHAP are well tolerated in spite of a few adverse events such as asthenia, diarrhoea, abdominal pain, anorexia, nausea, vomiting, headache and dizziness reported during different studies. These ADRs were not serious enough to discontinue anti-malarial treatments except for individuals in studies that reported serious adverse events such as jaundice, haemoglobulinuria, anaemia, convulsion, severe fatigue, severe malaria and deaths. In the current review, 3 study participant died during treatment [26]. This rate of mortality was not related to the study drugs on patients included in the study with uncomplicated malaria. Similarly, systematic reviews and meta-analyses conducted in Ethiopia and Sudan reported similar adverse events when patients with uncomplicated falciparum malaria were administered ACTs $[35,36,47]$.

\section{Strengths And Limitations Of The Study}

The current study has several strengths. A total of 39 studies with the same or different ACTs derived from 13 published articles and 3 unpublished studies were included that gave a total of 3,747 study participants. The study assessed efficacy of commonly used anti-malarial drugs: ASAQ and AL. Study outcomes were measured both clinically and parasitologically. Most studies evaluated the comparative efficacy of different anti-malarial medications.

However, the current study is not without limitations. The study included only mono-infection with $P$. falciparum with no available data on the other Plasmodium species. Moreover, not all studies reported AEs to anti-malarial drugs. Furthermore, there were fewer studies carried out in the Northern Regions of the country compared to the Southern Regions.

\section{Conclusion}

The present systematic review and meta-analyses reported a high overall efficacy of ACTs $(97.9 \%)$. The standard regimens, ASAQ, AL and DHAP showed high cure rates of $97.5 \%, 99.4 \%$ and $98.0 \%$ respectively. A number of adverse drug events were encountered such as asthenia, diarrhoea, abdominal pain, anorexia, nausea, vomiting, headache and dizziness after the administration of ACTs. However, the ADRs were not serious enough to discontinue the use of anti-malarial treatment except for a few patients who experienced severe adverse events. ACTs in Cameroon are still efficacious and well tolerated albeit with a slight decline in the efficacies of ASAQ and AL over time. There is need for continuous monitoring of 
efficacy of ACTs despite the high success cure rates as resistance seems inevitable since cases of antimalarial drug resistance have been reported in some areas of the world.

\section{Abbreviations}

ACT

Artemisinin-based combination therapy, AL:Artemether-lumefantrine, ASAQ:Artesunate-amodiaquine, ASCD:Artesunate-chloguanil-dapsone. AS-MQ:Artesunate-Mefloquine, AS-SP:Artesunate-SulphadoxinePyrimethamine, DHAP:Dihydroartemisinin-Piperaquine, NMA:Network meta-analysis, PRISMA:Preferred reporting items for systematic reviews and meta-analyses, STROBE:Strengthening the reporting of observational studies in epidemiology, SP:Sulphadoxine-Pyrimethamine; SP-AQ:SulphadoxinePyrimethamine-Amodiaquine

\section{Declarations}

\section{Ethics approval and consent to participate}

Not applicable because this is a systematic review and meta-analysis.

\section{Consent for publication}

Not applicable.

\section{Availability of data and materials}

All data and materials used for the analysis of this systematic review and meta-analysis are included in this write-up and the additional documents.

\section{Competing interests}

The authors declare that they have no competing interests.

\section{Funding}

WFM was commissioned by the WHO Country Office to document a comprehensive review on the efficacy of ACTs in Cameroon since their use, with financial support from the Global Malaria Programme (GMP), World Health Organisation, Geneva, Switzerland. WFM, AMN, IMA and PTNN are supported by the MARCAD Consortium through the DELTAS Africa Initiative [grant \# DEL-15-010] to the University of Yaounde I. The DELTAS Africa Initiative is an independent funding scheme of the African Academy of 
Sciences (AAS)'s Alliance for Accelerating Excellence in Science in Africa (AESA) and supported by the New Partnership for Africa's Development Planning and Coordinating Agency (NEPAD Agency) with funding from the Wellcome Trust [grant \# 107741/A/15/Z] and the UK government.

\section{Authors' contributions}

WFM conceived the research and coordinated the study. AMN, PTNN and CMM conducted the literature search, assessed potentially relevant studies for inclusion into the review, assessed the methodological quality of the included studies, and independently extracted the data. AMN and PTNN performed the statistical analysis, drafted the manuscript, critically reviewed the manuscript, and wrote the final manuscript. AMN and PTNN assessed potentially relevant studies for inclusion into the review, assessed the methodological quality of the included studies, independently extracted the data, and critically reviewed the manuscript. The authors WFM, PMN, IMA, RA, MNM, CMM, DAF, BAT, MSE, RD, JPKC, JDB, $A A, E A, E T, R G F L, A T$ and $P R$ proof read the manuscript. All authors read and approved the final manuscript.

\section{Acknowledgements}

Not applicable.

\section{References}

1. World Health Organisation (WHO). World Malaria Report 2019. https://www.who.int/publicationsdetail/world-malaria-report-2019. Accessed on 8th December, 2019.

2. Mbacham WF, Evehe M-SB, Netongo PM, Ateh IA, Mimche PN, Ajua A, et al. Efficacy of amodiaquine, sulphadoxine-pyrimethamine and their combination for the treatment of uncomplicated Plasmodium falciparum malaria in children in Cameroon at the time of policy change to artemisinin-based combination therapy. Malar J [Internet]. 2010;9:34-34. Available from: http://www.ncbi.nlm.nih.gov/pmc/articles/PMC2831903/.

3. 10.1186/1475-2875-11-113

Menard S, Morlais I, Tahar R, Sayang C, Mayengue P, Iriart X, et al. Molecular monitoring of plasmodium falciparum drug susceptibility at the time of the introduction of artemisinin-based combination therapy in Yaoundé, Cameroon: Implications for the future. Malar J [Internet]. 2012 [cited 2019 Jun 5];11(1):113. Available from:

http://malariajournal.biomedcentral.com/articles/10.1186/1475-2875-11-113.

4. $10.1186 / 1475-2875-6-146$

Kabanywanyi AM, Mwita A, Sumari D, Mandike R, Mugittu K, Abdulla S. Efficacy and safety of artemisinin-based antimalarial in the treatment of uncomplicated malaria in children in southern 
Tanzania. Malar J [Internet]. 2007 Nov 11;6(1):146. Available from: https://doi.org/10.1186/14752875-6-146.

5. Emergence and spread of

artemisinin resistance calls for intensified efforts to withdraw oral artemisinin

based monotherapy from the market (

http://aac.asm.lookup/doi/10.1128/AAC.01346-15

Emergence and spread of artemisinin resistance calls for intensified efforts to withdraw oral artemisinin-based monotherapy from the market (2014).

http://www.who.int/malaria/publications/atoz/policy-brief-withdrawal-of-oral-artemisinin-basedmonotherapies/en/. Accessed on 2nd December, 2016.6. Boussaroque A, Fall B, Madamet M, Camara C, Benoit N, Fall M, et al. Emergence of Mutations in the K13 Propeller Gene of Plasmodium falciparum Isolates from Dakar, Senegal, in 2013-2014. Antimicrob Agents Chemother [Internet]. 2016 Jan [cited 2019 Feb 10];60(1):624-7. Available from:

http://aac.asm.org/lookup/doi/10.1128/AAC.01346-15.

6. World Health Organisation (WHO). Guidelines for the treatment of malaria. Third edition. April 2015. https://www.who.int/malaria/publications/atoz/9789241549127/en/. Accessed on 15th July, 2020.

7. $10.1371 /$ journal.pmed.0050226

Okell LC, Drakeley CJ, Bousema T, Whitty CJM, Ghani AC. Modelling the Impact of Artemisinin Combination Therapy and Long-Acting Treatments on Malaria Transmission Intensity. PLOS Med [Internet]. 2008 Nov 25;5(11):e226. Available from: https://doi.org/10.1371/journal.pmed.0050226.

8. World Health Organisation (WHO). World Malaria Report 2008.

http://www.who.int/malaria/publications/atoz/9789241563697/en/. Accessed on 10th August, 2017.

9. Sayang C, Gausseres M, Vernazza-Licht N, Malvy D, Bley D, Millet P. Treatment of malaria from monotherapy to artemisinin-based combination therapy by health professionals in urban health facilities in Yaoundé, central province, Cameroon. Malar J. 2009;8(1):176.

10. Whegang Youdom S, Chiabi A, Basco LK. Monitoring the Efficacy and Safety of Artemisinin-Based Combination Therapies: A Review and Network Meta-analysis of Antimalarial Therapeutic Efficacy Trials in Cameroon. Drugs RD [Internet]. 2019 Mar;19(1):1-14. Available from: https://pubmed.ncbi.nlm.nih.gov/30656608.

11. Whegang Youdom S, Tahar R, Basco LK. Comparison of anti-malarial drug efficacy in the treatment of uncomplicated malaria in African children and adults using network meta-analysis. Malar $\mathrm{J}$ [Internet]. 2017;16:311. Available from: http://www.ncbi.nlm.nih.gov/pmc/articles/PMC5543626/.

12. Boni MF, Smith DL, Laxminarayan R. Benefits of using multiple first-line therapies against malaria. Proc Natl Acad Sci [Internet]. 2008 Sep 16;105(37):14216. Available from: http://www.pnas.org/content/105/37/14216.abstract.

13. The PRISMA Group 
10.1371/journal.pmed.1000097

Moher D, Liberati A, Tetzlaff J, Altman DG. The PRISMA Group. Preferred Reporting Items for Systematic Reviews and Meta-Analyses: The PRISMA Statement. PLOS Med [Internet]. 2009 Jul 21;6(7):e1000097. Available from: https://doi.org/10.1371/journal.pmed.1000097.

14. 10.1371/journal.pmed.0040296

Jadad AR, Moore RA, Carroll D, Jenkinson C, Reynolds DJ, Gavaghan DJ, et al. Assessing the quality of reports of randomized clinical trials: is blinding necessary? Control Clin Trials. 1996 Feb;17(1):112. 16. von Elm E, Altman DG, Egger M, Pocock SJ, Gøtzsche PC, Vandenbroucke JP, et al. The Strengthening the Reporting of Observational Studies in Epidemiology (STROBE) Statement: Guidelines for Reporting Observational Studies. PLOS Med [Internet]. 2007 Oct 16;4(10):e296. Available from: https://doi.org/10.1371/journal.pmed.0040296.

15. World Health Organisation. Division of Control of Tropical Diseases. (1996). Assessment of therapeutic efficacy of ant. Assessment of therapeutic efficacy of antimalarial drugs: for uncomplicated falciparum malaria in areas with intense transmission. Geneva : World Health Organization. http://www.who.int/iris/handle/10665/63295. Accessed on 12th February, 2019.18. World Health Organisation. Cluster on Communicable Diseases \& WHO Consultation on Monitoring Antimalarial Drug Resistance. (2002). Monitoring antimalarial drug resistance: report of a WHO consultation, Geneva, Switzerland, 3-5 December 2001. Geneva: World Health Organization. http://www.who.int/iris/handle/10665/67590. Accessed on 12th February, 2019.

16. WHO. Assessment and monitoring of antimalarial drug efficacy for the treatment of uncomplicated falciparum malaria (archived). December 2003. https://www.who.int/malaria/publications/atoz/whohtmrbm200350/en/. Accessed on 12th February, 2019.

17. WHO. Methods for surveillance of antimalarial drug efficacy. November 2009. https://www.who.int/malaria/publications/ato.. https://www.who.int/malaria/publications/atoz/9789241597531/en/. Accessed on 12th February, 2019.

18. Cochrane Consumers and Communication Review Group. Heterogeneity and subgroup analyses in Cochrane Consumers and Communication Group reviews: planning the analysis at protocol stage. http://cccrg.cochrane.org. Accessed on 12th February, 2019.22

10.1186/1475-2875-10-185

Ryan R, Cochrane Consumers and Communication Review Group. Heterogeneity and subgroup analyses in Cochrane Consumers and Communication Group reviews: planning the analysis at protocol stage. http://cccrg.cochrane.org. Accessed on 12th February, 2019.22. Menan H, Faye O, Same-Ekobo A, Oga ASS, Faye B, Kiki Barro CP, et al. Comparative study of the efficacy and tolerability of dihydroartemisinin - piperaquine - trimethoprim versus artemether - lumefantrine in the treatment of uncomplicated Plasmodium falciparum malaria in Cameroon, Ivory Coast and Senegal. Malar J [Internet]. 2011 Jul 8;10(1):185. Available from: https://doi.org/10.1186/1475-2875-10-185. 
19. Sagara I, Rulisa S, Mbacham W, Adam I, Sissoko K, Maiga H, et al. Efficacy and safety of a fixed dose artesunate-sulphamethoxypyrazine-pyrimethamine compared to artemether-lumefantrine for the treatment of uncomplicated falciparum malaria across Africa: a randomized multi-centre trial. Malar J [Internet]. 2009 Apr 14;8:63-63. Available from: https://www.ncbi.nlm.nih.gov/pubmed/19366448.

20. Faye B, Kuété T, Kiki-Barro CP, Tine RC, Nkoa T, Ndiaye JLA, et al. Multicentre study evaluating the non-inferiority of the new paediatric formulation of artesunate/amodiaquine versus artemether/lumefantrine for the management of uncomplicated Plasmodium falciparum malaria in children in Cameroon, Ivory Coast and Senegal. Malar J [Internet]. 2012 Dec 27;11:433-433. Available from: https://www.ncbi.nlm.nih.gov/pubmed/23270636.

21. $10.1093 /$ infdis/jiu341

Tahar R, Almelli T, Debue C, Foumane Ngane V, Djaman Allico J, Whegang Youdom S, et al. Randomized Trial of Artesunate-Amodiaquine, Atovaquone-Proguanil, and Artesunate-AtovaquoneProguanil for the Treatment of Uncomplicated Falciparum Malaria in Children. J Infect Dis [Internet]. 2014 Dec [cited 2018 Nov 15];210(12):1962-71. Available from:

https://academic.oup.com/jid/article-lookup/doi/10.1093/infdis/jiu341.

22. Ndiaye JL, Randrianarivelojosia M, Sagara I, Brasseur P, Ndiaye I, Faye B, et al. Randomized, multicentre assessment of the efficacy and safety of ASAQ - a fixed-dose artesunate-amodiaquine combination therapy in the treatment of uncomplicated Plasmodium falciparum malaria. Malar $\mathrm{J}$ [Internet]. 2009;8:125-125. Available from:

http://www.ncbi.nlm.nih.gov/pmc/articles/PMC2698916/.

23. Whegang SY, Tahar R, Foumane VN, Soula G, Gwét H, Thalabard J-C, et al. Efficacy of nonartemisinin- and artemisinin-based combination therapies for uncomplicated falciparum malaria in Cameroon. Malar J [Internet]. 2010;9:56-56. Available from:

http://www.ncbi.nlm.nih.gov/pmc/articles/PMC2834703/.

24. Yavo W, Faye B, Kuete T, Djohan V, Oga SA, Kassi RR, et al. Multicentric assessment of the efficacy and tolerability of dihydroartemisinin-piperaquine compared to artemether-lumefantrine in the treatment of uncomplicated Plasmodium falciparum malaria in sub-Saharan Africa. Malar $\mathrm{J}$ [Internet]. 2011 Jul 20;10:198-198. Available from:

https://www.ncbi.nlm.nih.gov/pubmed/21774826.

25. 10.1186/1475-2875-7-16

Ndiaye JLA, Faye B, Diouf AM, Kuété T, Cisse M, Seck PA, et al. Randomized, comparative study of the efficacy and safety of artesunate plus amodiaquine, administered as a single daily intake versus two daily intakes in the treatment of uncomplicated falciparum malaria. Malar J [Internet]. $2008 \mathrm{Jan}$ 19;7(1):16. Available from: https://doi.org/10.1186/1475-2875-7-16.

26. Nji AM, Ali IM, Moyeh MN, Ngongang E-O, Ekollo AM, Chedjou J-P, et al. Randomized non-inferiority and safety trial of dihydroartemisin-piperaquine and artesunate-amodiaquine versus artemetherlumefantrine in the treatment of uncomplicated Plasmodium falciparum malaria in Cameroonian children. Malar J [Internet]. 2015;14:27. Available from: http://www.ncbi.nlm.nih.gov/pmc/articles/PMC4318246/. 
27. Kimbi HK, Ntoko M, Ntonifor NN, Lum E, Njunda AL, Fon PN. Efficacy and Tolerability of Malartin and Sulphadoxine-Pyrimethamine Combination against Uncomplicated Falciparum Malaria in Dibanda, Southwest Cameroon. J Trop Med [Internet]. 2012 [cited 2019 Feb 13];2012:1-7. Available from: http://www.hindawi.com/journals/jtm/2012/372518/.

28. Tietche F, Chelo D, Mina Ntoto NK, Djoukoue FM, Hatz C, Frey S, et al. Tolerability and efficacy of a pediatric granule formulation of artesunate-mefloquine in young children from Cameroon with uncomplicated falciparum malaria. Am J Trop Med Hyg [Internet]. 2010 Jun;82(6):1034-40. Available from: https://www.ncbi.nlm.nih.gov/pubmed/20519597.

29. Apinjoh T, Anchang-Kimbi J, Ajonina M, Njonguo E, Njua-Yafi C, Ngwai A, et al. In Vivo Efficacy of Artesunate/Sulphadoxine-Pyrimethamine versus Artesunate/Amodiaquine in the Treatment of Uncomplicated P. falciparium Malaria in Children around the Slope of Mount Cameroon: A Randomized Controlled Trial. Biomedicines [Internet]. 2016 Feb 15 [cited 2018 Nov 16];4(1):5. Available from: http://www.mdpi.com/2227-9059/4/1/5.

30. $10.1179 / 136485907 X 156942$

Kimbi HK, Nkuo-Akenji TK, Patchong AFM, Ndamukong KN, Nkwescheu A. The comparative efficacies of malartin, with and without amodiaquine, in the treatment of Plasmodium falciparum malaria in the Buea district of Cameroon. Ann Trop Med Parasitol [Internet]. 2007 Mar [cited 2018 Nov 15];101(2):95-102. Available from:

http://www.tandfonline.com/doi/full/10.1179/136485907X156942.

31. $10.1186 / \mathrm{s} 12936-018-2265-\mathrm{x}$

Adam I, Ibrahim Y, Gasim Gl. Efficacy and safety of artemisinin-based combination therapy for uncomplicated Plasmodium falciparum malaria in Sudan: a systematic review and meta-analysis. Malar J [Internet]. 2018 Dec [cited 2019 May 19];17(1). Available from: https://malariajournal.biomedcentral.com/articles/10.1186/s12936-018-2265-x.

32. $10.1186 / \mathrm{s} 12936-017-1922-9$

Gebreyohannes EA, Bhagavathula AS, Seid MA, Tegegn HG. Anti-malarial treatment outcomes in Ethiopia: a systematic review and meta-analysis. Malar J [Internet]. 2017 Dec [cited 2019 May 19];16(1). Available from: http://malariajournal.biomedcentral.com/articles/10.1186/s12936-0171922-9.

33. Basco LK. Molecular epidemiology of malaria in Cameroon. XIII. Analysis of pfcrt mutations and in vitro chloroquine resistance. Am J Trop Med Hyg. 2002 Oct;67(4):388-91.

34. Basco LK, Ndounga M, Ngane VF, Soula G. Molecular epidemiology of malaria in Cameroon. XIV. Plasmodium falciparum chloroquine resistance transporter (PFCRT) gene sequences of isolates before and after chloroquine treatment. Am J Trop Med Hyg. 2002 Oct;67(4):392-5.

35. Basco LK, Same-Ekobo A, Ngane VF, Ndounga M, Metoh T, Ringwald P, et al. Therapeutic efficacy of sulfadoxine-pyrimethamine, amodiaquine and the sulfadoxine-pyrimethamine-amodiaquine combination against uncomplicated Plasmodium falciparum malaria in young children in Cameroon. Bull World Health Organ [Internet]. 2002;80(7):538-45. Available from: http://www.ncbi.nlm.nih.gov/pmc/articles/PMC2567565/. 
36. $10.1186 / 1475-2875-8-203$

Zwang J, Olliaro P, Barennes H, Bonnet M, Brasseur P, Bukirwa $\mathrm{H}$, et al. Efficacy of artesunateamodiaquine for treating uncomplicated falciparum malaria in sub-Saharan Africa: a multi-centre analysis. Malar J [Internet]. 2009 Dec [cited 2019 May 18];8(1). Available from: https://malariajournal.biomedcentral.com/articles/10.1186/1475-2875-8-203.

37. 10.4269/ajtmh.2011.11-0069

Cousin M, Hunt P, Walter V, Rosenthal PJ, Falade CO, Premji ZG, et al. Efficacy and Safety of Artemether-Lumefantrine in the Treatment of Acute, Uncomplicated Plasmodium falciparum Malaria: A Pooled Analysis. Am J Trop Med Hyg [Internet]. 2011 Nov 1 [cited 2019 May 18];85(5):793-804. Available from: http://www.ajtmh.org/content/journals/10.4269/ajtmh.2011.11-0069.

38. Naing C, Whittaker MA, Htet NH, Aye SN, Mak JW. Efficacy of antimalarial drugs for treatment of uncomplicated falciparum malaria in Asian region: A network meta-analysis. PloS One [Internet]. 2019 Dec 19;14(12):e0225882-e0225882. Available from:

https://pubmed.ncbi.nlm.nih.gov/31856172.

39. Sevene E, Banda CG, Mukaka M, Maculuve S, Macuacua S, Vala A, et al. Efficacy and safety of dihydroartemisinin-piperaquine for treatment of Plasmodium falciparum uncomplicated malaria in adult patients on antiretroviral therapy in Malawi and Mozambique: an open label non-randomized interventional trial. Malar J [Internet]. 2019 Aug 20;18(1):277-277. Available from: https://pubmed.ncbi.nlm.nih.gov/31429785.

40. National Malaria Control Programme. (2013): Guidelines for the Management of Malaria in Cameroon.

41. WWARN Artemisinin based Combination Therapy (ACT) Africa Baseline Study Group WWARN Artemisinin based Combination Therapy (ACT) Africa Baseline Study Group. Dahal P, d'Alessandro U, Dorsey G, Guerin PJ, Nsanzabana C, et al. Clinical determinants of early parasitological response to ACTs in African patients with uncomplicated falciparum malaria: a literature review and meta-analysis of individual patient data. BMC Med [Internet]. $2015 \mathrm{Sep}$ 7;13:212-212. Available from: https://pubmed.ncbi.nlm.nih.gov/26343145.

42. 10.1186/s40249-017-0350-y

Apinjoh TO, Mugri RN, Miotto O, Chi HF, Tata RB, Anchang-Kimbi JK, et al. Molecular markers for artemisinin and partner drug resistance in natural Plasmodium falciparum populations following increased insecticide treated net coverage along the slope of mount Cameroon: cross-sectional study. Infect Dis Poverty [Internet]. 2017 Dec [cited 2019 May 4];6(1). Available from: http://idpjournal.biomedcentral.com/articles/10.1186/s40249-017-0350-y.

43. Zwang J, Dorsey G, Djimdé A, Karema C, Mårtensson A, Ndiaye J-L, et al. Clinical tolerability of artesunate-amodiaquine versus comparator treatments for uncomplicated falciparum malaria: an individual-patient analysis of eight randomized controlled trials in sub-Saharan Africa. Malar $\mathrm{J}$ [Internet]. 2012 Aug 2;11:260-260. Available from: https://pubmed.ncbi.nlm.nih.gov/22856598.

\section{Additional files.}


45. Additional file 1. Summary characteristics of all studies of the systematic review and meta-analysis on efficacy and safety ACTs in Cameroon (included and unpublished studies).

46. Additional file 2. Assessment of publication bias on efficacy and safety of ACTs in Cameroon.

47. Additional file 3. Modified Jadad scale for study on efficacy and safety of ACTs in Cameroon.

48. Additional file 4. STROBE quality assessment for study on efficacy and safety of ACTs in Cameroon.

49. Additional file 5. Proportion of Fever Clearance and Adverse Events for ACTs in Cameroon.

\section{Figures}

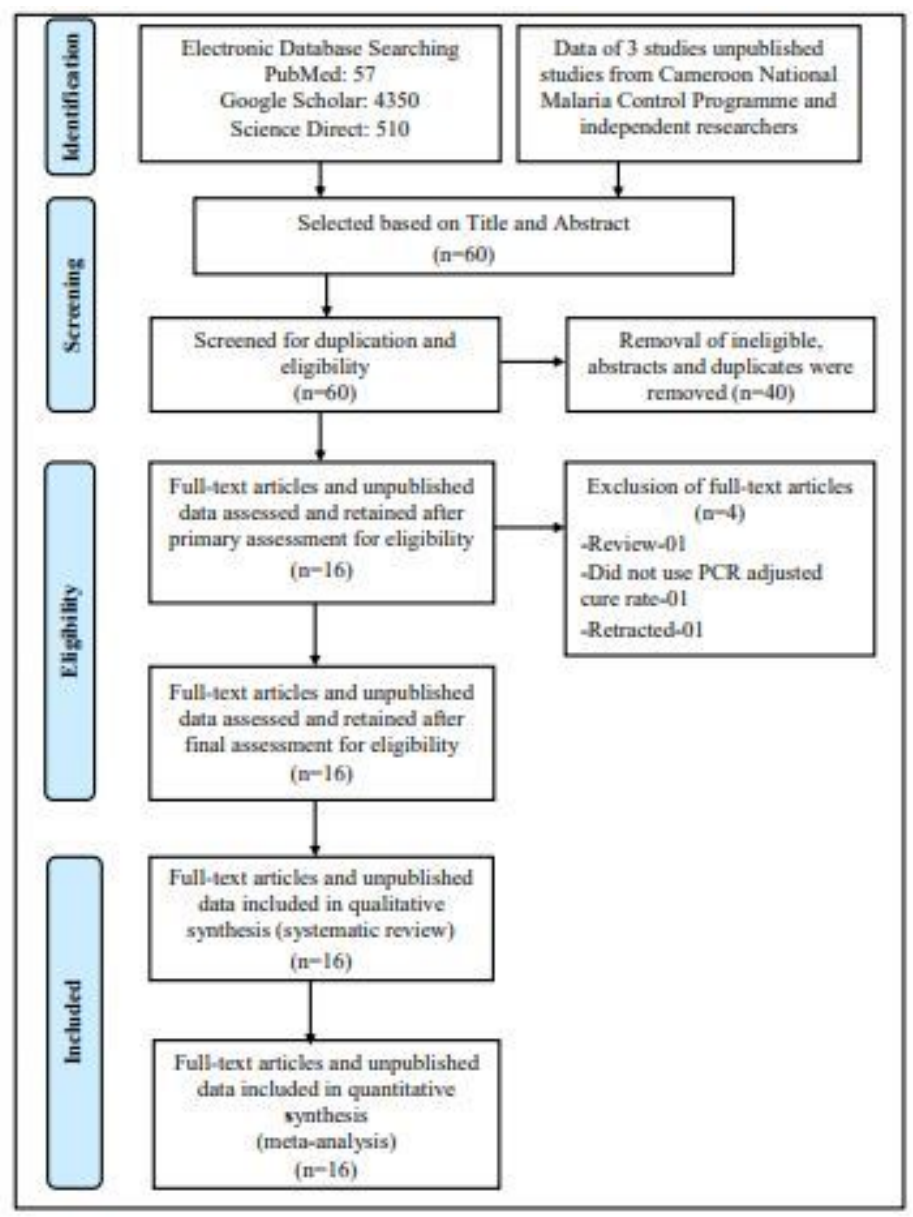

Figure 1

Flow chart showing the number of articles included in the systematic review and meta-analysis on artemisinin-based anti-malarial treatment outcomes in Cameroon 
Studily

Kimbi et al, 2007

Yauba et al, 2007

Yuba et al, 2007

hiba et al, 2007

Yauba ef al, 2007

Nolaye et al, 2008

Nchaye et al, 2008

Sagara et al, 2009

Sagara et al, 2009

Sagara et al, 2009

Ndiaye et al , 2009

Ndiaye et al, 2009

Nolaye et al, 2009

Whegang et al, 2010

Whegang et al, 2010

Whegang et al, 2010

Whegang et al, 2010

Whegang et al, 2010

Whegang et al, 2010

Whegang et al, 2010

Whegang et al, 2010

Whegang et al 2010

Yavo et al. 2011

Yavo et al, 2011

Menan et al, 2011

Menan et al, 2011

Kintri et al, 2012

Noono ef al, 2012

Faye et al, 2012

Tahar et al, 2014

Tahar ef al, 2014

Akindeh et al, 2015

Akindeh et al, 2015

Nkindeh et al, 2015

Apinioh et al, 2016

Apinioh et al, 2016

Ngwalor et al, 2016
Tietche et al, 2010

Faye et al, 2012

Efficacy of ACTS Totel samples Prevalence

$95 \% \mathrm{Cl}$

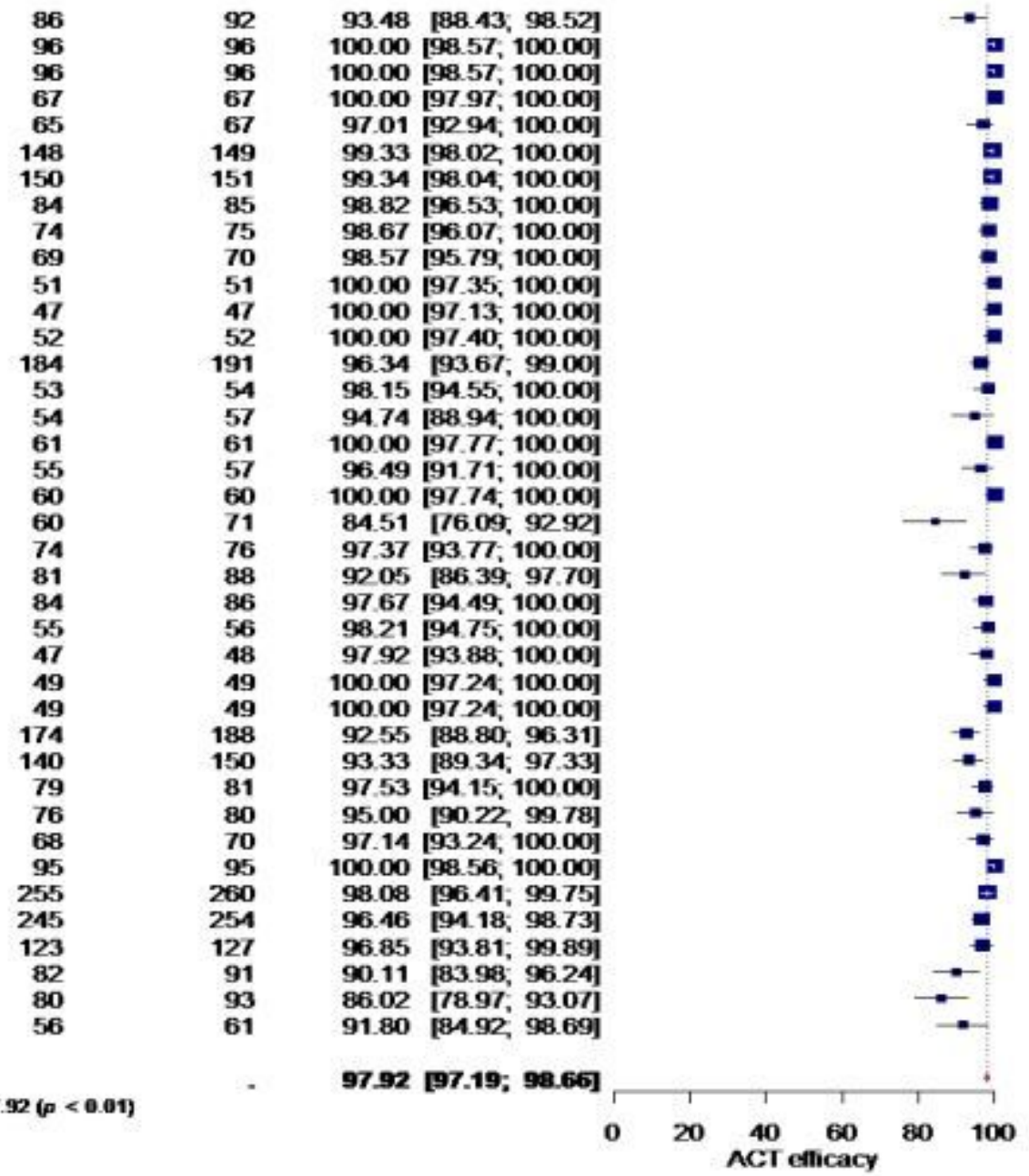

Figure 2
$93.48[38.43,98.52]$ $98.57[95.79,100.00$ 9474 [88.94; 10000 $97.37[93.77 ; 10000]$ $9205[86.39,97.70$ $194,49 \div 10000$ (88. 80; 96.31 $33[8934 ; 97,33$ $[94.15,10000]$ $96.85[93.81 ; 99.89$ 97.92 [97.19; 98.66$]$

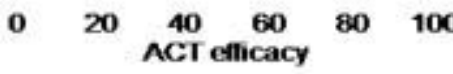
[98.04; 10000

Overall treatment success rate of artemisinin-based combination therapies (ACTs) published between 2004 and 2020 in Cameroon 
Study

Kimbi et al. 2007

Yauba et al., 2007

Yauba et al., 2007

Ndiaye et al, 2008

Ndiaye et al., 2008

Ndiaye et al, 2009

Ndiaye et al., 2009

Whegang et al, 2010

Whegang et al, 2010

Whegang et al., 2010

Ngono et al., 2012

Faye et al., 2012

Tahar et al., 2014

Akindeh et al., 2015

Apinjoh et al., 2016

Ngwafor et al, 2016

$95 \%$ C.I.

Random effects model

Heterogeneity: $I^{2}=60 \%, t^{2}=0.0004, x_{15}^{2}=37.66(p<0.01)$

93.48 [88.43; 98.52$]$

$100.00[98.57 ; 100.00]$

97.01 [92.94; 100.00]

$99.33[98.02 ; 100.00]$

$99.34[98.04 ; 100.00]$

$100.00[97.35 ; 100.00]$

$100.00[97.13,100.00]$

$98.15[94.55 ; 100.00]$

$96.49[91.71 ; 100.00]$

92.05 [86.39, 97.70]

$93.33[89.34 ; 97.33]$

$97.53[94.15,100.00]$

97.14 [93.24; 100.00]

98.08 [96.41; 99.75]

$90.11[83.98 ; 96.24]$

91.80 [84.92; 98.69]

97.54 [96.27; 98.81]

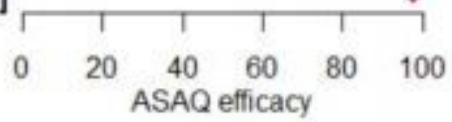

\section{Figure 3}

Cure rates of artesunate-amodiaquine (AS-AQ) published from 2004 to 2020 in Cameroon

Study

Yauba et al, 2007

Yauba et al., 2007

Sagara et al., 2009

Ndiaye et al., 2009

Whegang et al., 2010

Yavo et al., 2011

Menan et al., 2011

Faye et al, 2012

Akindeh et al., 2015
Efficacy of AL Total samples Prevalence

$95 \%$ C.I.

$\begin{array}{rrrr}96 & 96 & 100.00[98.57 ; 100.00] \\ 67 & 67 & 100.00[97.97 ; 100.00] \\ 69 & 70 & 98.57 & {[95.79 ; 100.00]} \\ 52 & 52 & 100.00[97.40 ; 100.00] \\ 60 & 60 & 100.00[97.74 ; 100.00] \\ 47 & 48 & 97.92[93.88 ; 100.00] \\ 49 & 49 & 100.00[97.24 ; 100.00] \\ 76 & 80 & 95.00 & {[90.22 ; 99.78]} \\ 123 & 127 & 96.85 & {[93.81 ; 99.89]}\end{array}$

Random effects model

Heterogeneity: $I^{2}=6 \%, t^{2}<0.0001, x_{4}^{2}=8.49(p=0.39)$
$99.42[98.61 ; 100.00]$

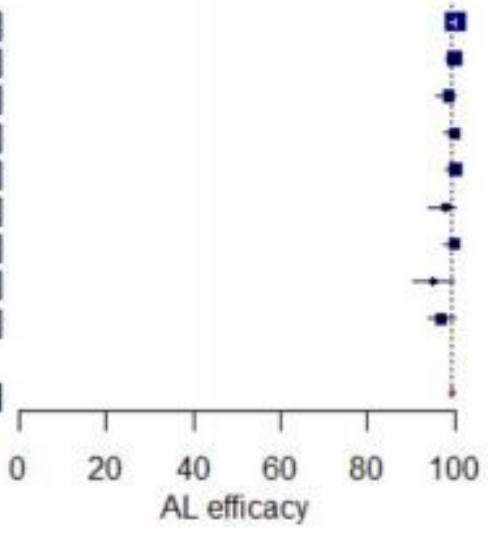

\section{Figure 4}

Adequate clinical parasitological response of artemether-lumefantrine (AL) published between 2004 and 2020 in Cameroon 
Study

Whegang et al., 2010

Yavo et al., 2011

Menan et al., 2011

Akindeh et al., 2015
Efficacy of DHAP Total samples Prevalence $\quad 95 \%$ C.I.

$84 \quad 86 \quad 97.67[94.49 ; 100.00]$

$55 \quad 56 \quad 98.21[94.75 ; 100.00]$

$49 \quad 49 \quad 100.00[97.24 ; 100.00]$

$245 \quad 254 \quad 96.46[94.18 ; 98.73]$

\section{Random effects model}

Heterogeneity: $I^{2}=22 \%, \tau^{2}<0.0001, \gamma_{3}^{2}=3.83(p=0.28)$

97.97 [96.28; 99.67]

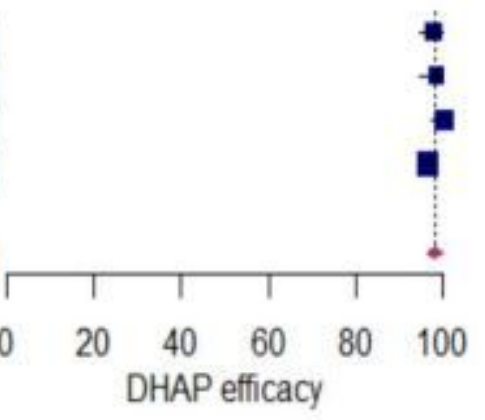

\section{Figure 5}

Treatment success rate of dihydroartemisinin-piperaquine (DHAP) published between 2004 and 2020 in Cameroon

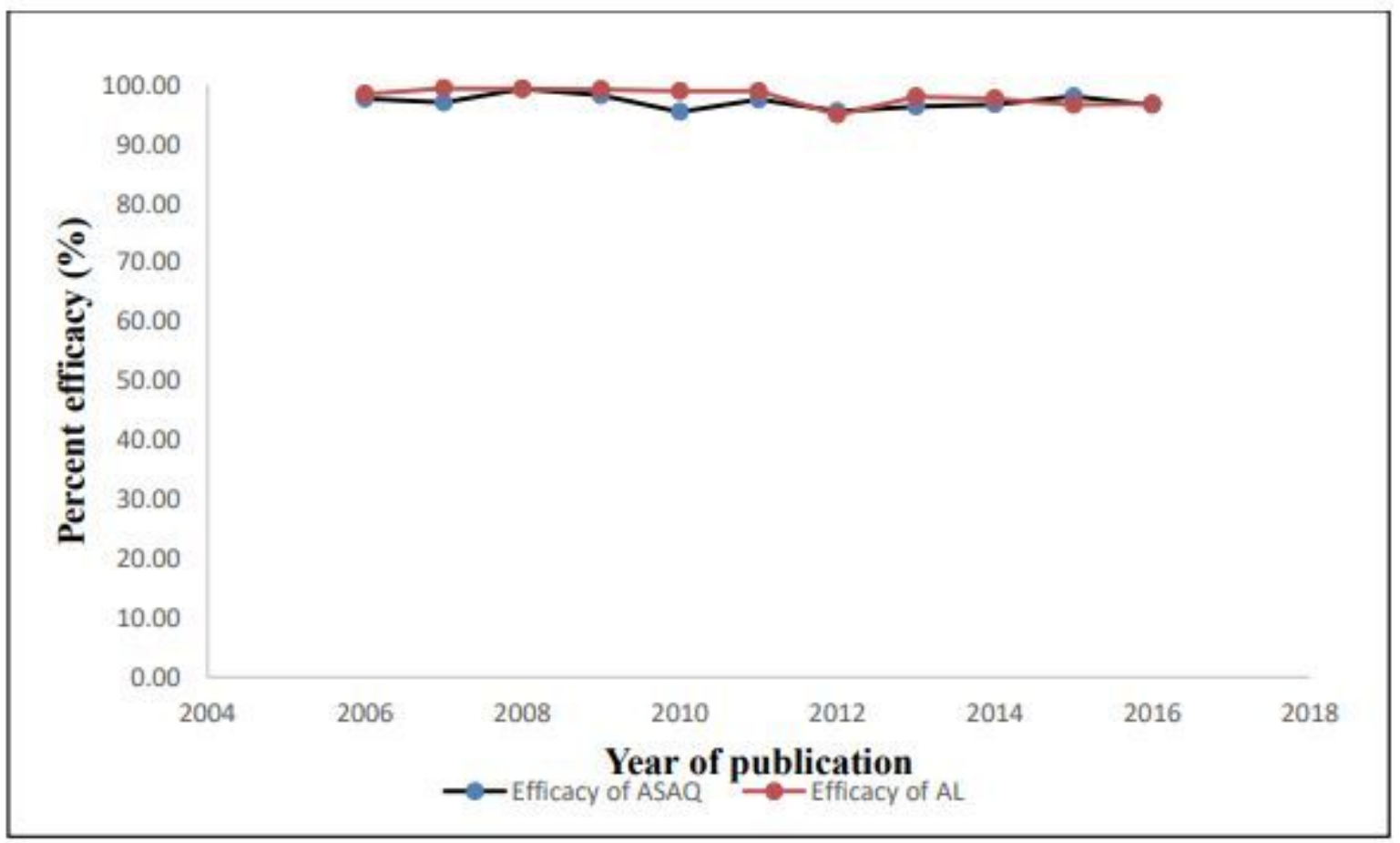

\section{Figure 6}

Evolution in the efficacies of ASAQ and AL from 2006 to 2018 


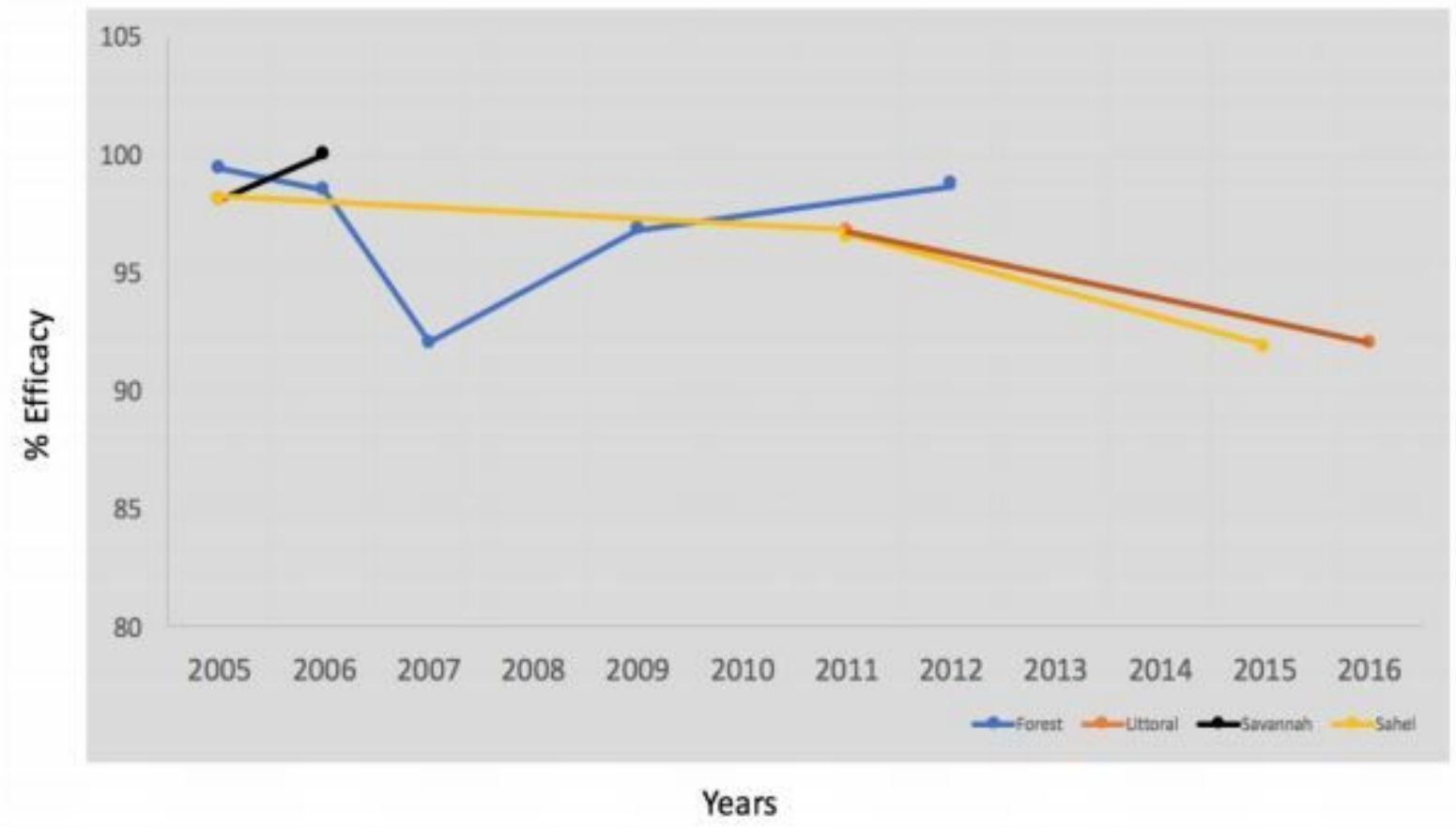

Figure 7

Evolution of Clinical Efficacy of ASAQ per Ecological Zones of Cameron

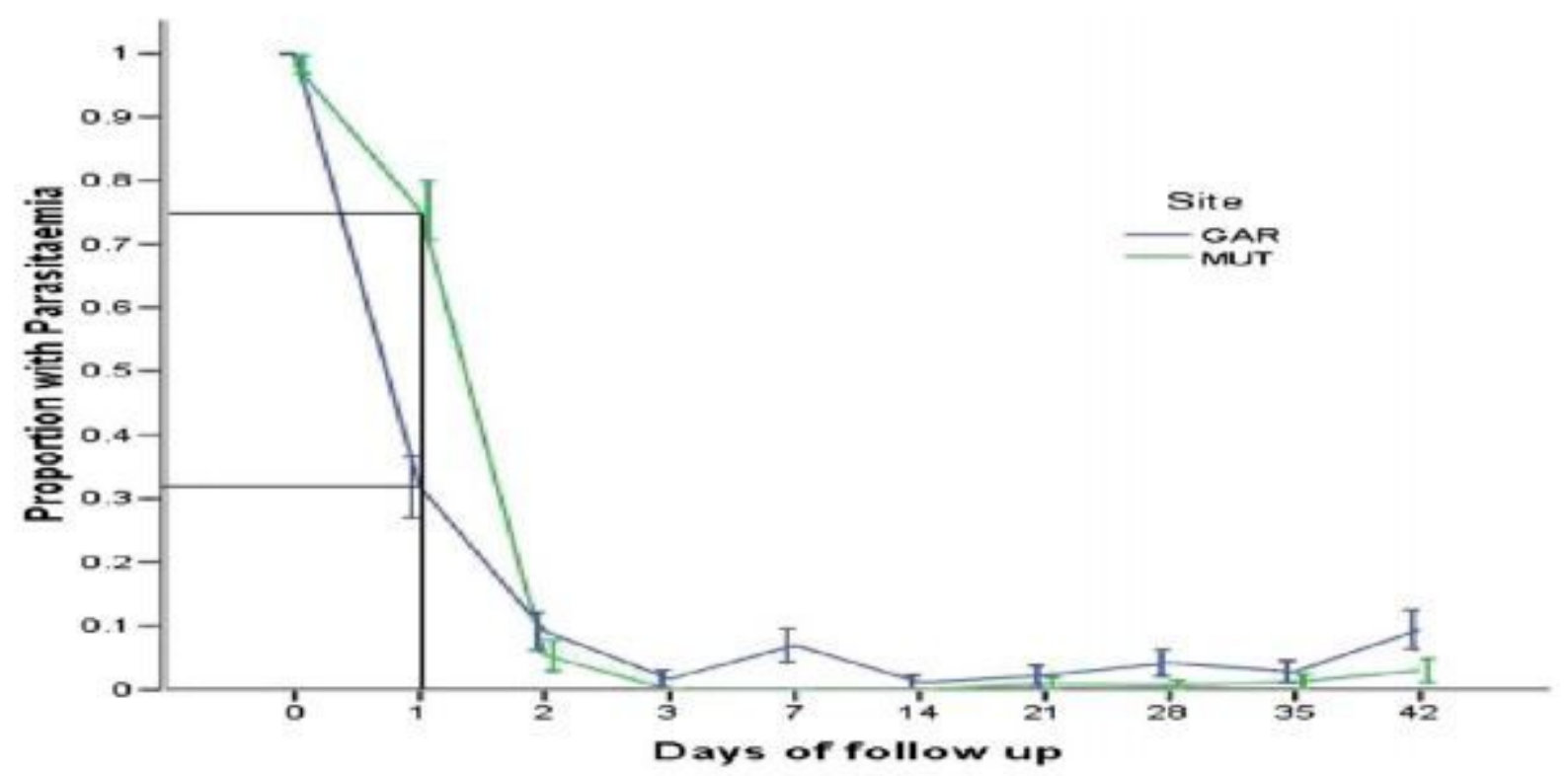

Figure 8

Parasite Clearance with ACTs in Garoua and Mutengene GAR-Garoua and MUT-Mutengene. Delayed parasite clearance was observed among most study participants in Mutengene on D1 post-treatment. 


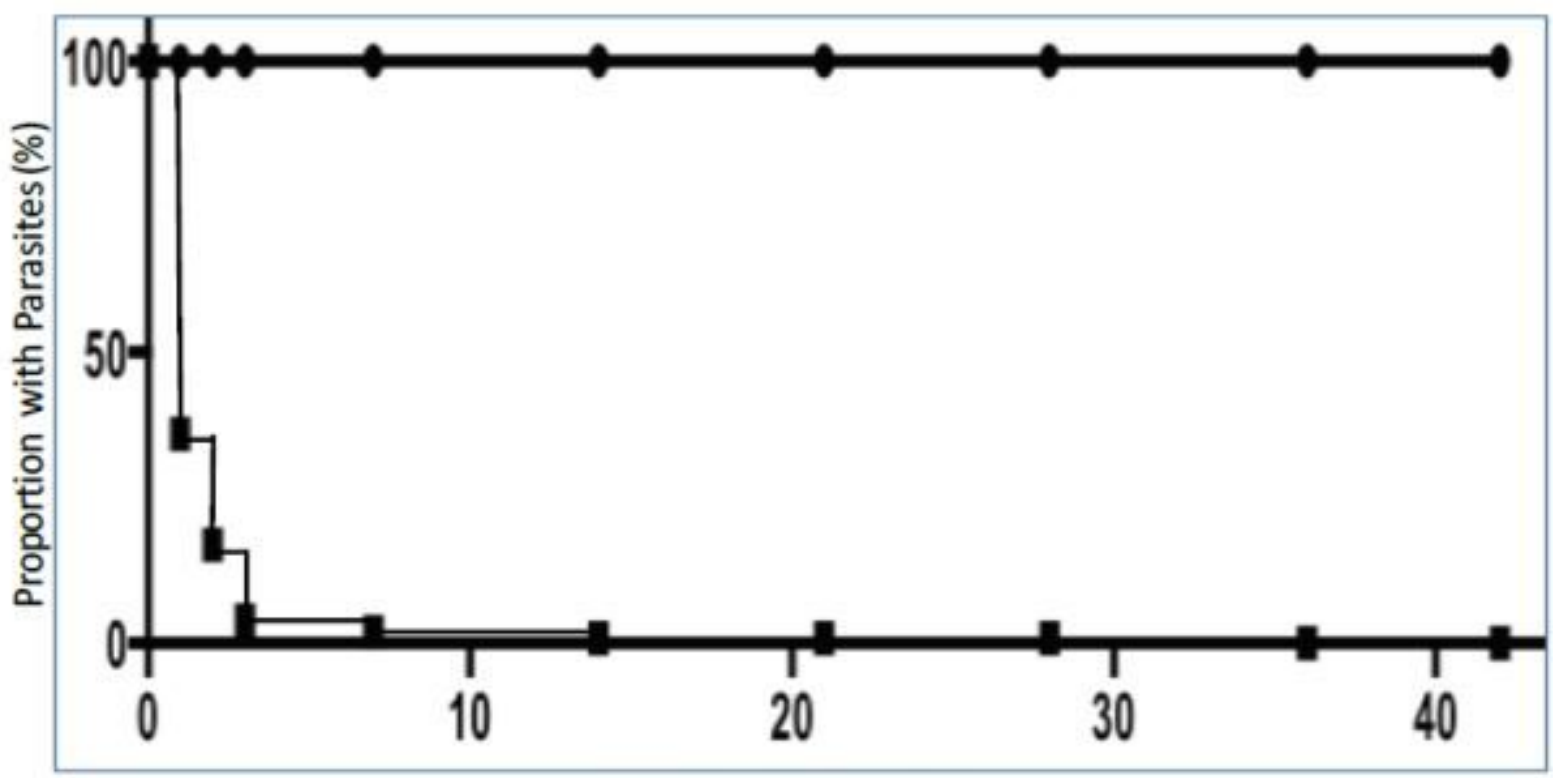

Days Post Therapy

Figure 9

Kaplan-Meier Analysis for Parasite Clearance of ACTs from 2004-2020 The average parasite clearance did not take into account the two studies with outlier figures. In addition, the circles represent the hypothetical situation of no clearance and the squares represent clearance by all ACTs.

\section{Supplementary Files}

This is a list of supplementary files associated with this preprint. Click to download.

- Additionalfile5.xlsx

- Additionalfile4.xlsx

- Additionalfile3.xlsx

- Additionalfile2.docx

- Additionalfile1.xlsx

- MonitoringofACTsinCMR.pdf

- PersonalcoverforthestudyonACTsinCMR.pdf 